\title{
Reanalysis of Experiments To Quantify Irreversibility of Pesticide Sorption-Desorption in Soil
}

\author{
Laura A Suddaby \\ Environment Department, University of York, Heslington, York, YO10 5DD, U.K.
}

Sabine Beulke

Food and Environment Research Agency, Sand Hutton, York, YO41 1LZ, U.K.

Wendy van Beinum

Food and Environment Research Agency, Sand Hutton, York, YO41 1LZ, U.K.

\section{Rafael Celis}

Institute for Natural Resources and Agrobiology of Seville (IRNAS), Science Research Council (CSIC), Av. Reina Mercedes 10, 41012 Seville, Spain

\section{William C. Koskinen}

Soil and Water Management Research Unit, Agricultural Research Service, U.S. Department of Agriculture, 1991 Upper Buford Circle, Room 439, University of Minnesota, St. Paul, Minnesota 55108, United States

\section{Colin D. Brown}

Environment Department, University of York, Heslington, York, YO10 5DD, U.K.

\begin{abstract}
Previously published research used an isotope-exchange technique to measure irreversibility of pesticide sorption-desorption in soil. Results indicated significant irreversibility (6-51\%) in sorption in five pesticide-soil systems measured over $72 \mathrm{~h}$. Here, we propose a three-site model to reanalyze the experimental data. The model adds a slow but reversible binding on nonequilibrium sorption sites in addition to instantaneously reversible sites and irreversible sites. The model was able to match experimental data very closely, but only if irreversible sorption was assumed to be absent. Observed asymmetry in the binding of ${ }^{12} \mathrm{C}$ - and ${ }^{14} \mathrm{C}$-pesticide was explained on the basis of nonattainment of sorption equilibrium over the study period. Results suggest that irreversible sorption may be less significant than previously considered with important implications for understanding the fate of pesticides applied to soil.
\end{abstract}

KEYWORDS: irreversible sorption, three-site model, pesticide, soil, isotope exchange

\section{INTRODUCTION}

Accurate prediction of the behavior of pesticides in the environment relies on robust understanding of their interactions with soil. Sorption is a key process in soil that influences pesticide efficacy as well as availability for runoff, leaching, degradation, and uptake into nontarget organisms. ${ }^{1-3}$ Sorption determines solute distribution between soil and water, whereas desorption controls reversibility of the initial binding. ${ }^{4}$ Some evidence suggests that sorption occurs with only partial reversibility and that a proportion of sorbed pesticide binds irreversibly to the soil matrix. ${ }^{s-7}$ The study of irreversible sorption has been confounded by use of harsh soil extraction techniques and procedural difficulties involved in distinguishing between slowly reversible and irreversible sorption. ${ }^{8,9}$
Celis and Koskinen ${ }^{10_{1} 11}$ used an isotope-exchange technique to assess the reversibility of pesticide sorption to soil in situ. The method leaves the soil matrix essentially unchanged as the composition of the soil solution is not disturbed. Thus, it is a relatively noninvasive method by which irreversibility in the sorption-desorption process may be assessed. Sander and Pignatello ${ }^{12}$ also propose that isotope exchange is a promising method to unequivocally establish whether sorbate entrapment occurs during a sorption-desorption cycle. The approach relies 
on establishing sorption equilibrium during a sorption phase, then determining the proportion of sorbed pesticide residue that participates in subsequent exchange with a second isotope of the same pesticide. Time-dependent but reversible sorption is a potential confounder of the approach. ${ }^{10,13}$ Here, we describe a three-site sorption model to incorporate this component and use it to re-evaluate isotope-exchange experiments seeking to quantify irreversibility in pesticide sorption to soil.

\section{ISOTOPE-EXCHANGE EXPERIMENTS}

Celis and Koskinen's ${ }^{10,11}$ method involves preparation of two tubes (A and B) for each equilibrium point of their sorption isotherm. Tubes A and $B$ contain identical amounts of soil, solution, and initial pesticide concentration, but tube $\mathrm{A}$ contains only ${ }^{12} \mathrm{C}$-pesticide whereas tube $\mathrm{B}$ contains both ${ }^{12} \mathrm{C}$ - and ${ }^{14} \mathrm{C}$-labeled pesticide. The suspensions are shaken at room temperature for $24 \mathrm{~h}$ (adsorption period). After centrifuging, the supernatant of tube $\mathrm{A}\left({ }^{12} \mathrm{C}\right)$ is replaced with the supernatant of tube $\mathrm{B}\left({ }^{12} \mathrm{C}+{ }^{14} \mathrm{C}\right)$ and vice versa. It is assumed that sorption equilibrium is not disturbed because the same equilibrium should be reached in both tubes, albeit with different carbon isotopes. The subsequent exchange between ${ }^{12} \mathrm{C}$ - and ${ }^{14} \mathrm{C}$-pesticide in tubes $\mathrm{A}$ and $\mathrm{B}$ is then observed during a $48 \mathrm{~h}$ re-equilibration time (sampling at $1,7,24,48 \mathrm{~h}$ ). This characterizes the kinetics of pesticide exchange and provides an estimate of the amount of sorbed pesticide that did not participate in the exchange.

In their first paper, Celis and Koskinen ${ }^{10}$ studied isotope exchange of the pesticides triadimefon and imidacloprid-guanidine to Hanford (fine sandy loam) and Drummer (silty clay loam) soils. They reported that their two-compartment model showed that approximately $90 \%$ of sorption of triadimefon and imidacloprid-guanidine to the Hanford soil occurred on reversible, easily desorbable sites and $10 \%$ of the sorbed molecules were irreversibly sorbed. Celis and Koskinen ${ }^{11}$ went on to study the isotope exchange of imidacloprid and imidaclopridurea to a silty clay loam ( $\mathrm{SiCL}$ ), and that of imidacloprid-guanidine to a loamy sand (LS). The authors applied the same two-compartment model in their second paper and reached a similar conclusion, reporting that the percentage of irreversibly sorbed pesticide increased in the order imidacloprid-SiCL soil $(6-32 \%)<$ imidacloprid ureaSiCL soil $(15-23 \%)<$ imidacloprid guanidine-LS soil $(32-51 \%)$.

Celis and Koskinen ${ }^{10,11}$ observed unusual behavior in their results, with asymmetry in the sorption patterns for initially ${ }^{12} \mathrm{C}$ (tube A) and ${ }^{14} \mathrm{C}$ (tube B) samples. Desorption of ${ }^{14} \mathrm{C}$-pesticide (tube B) was slower than the sorption of ${ }^{14} \mathrm{C}$-pesticide (tube $\mathrm{A}$ ), which suggests that true equilibrium had not been attained and so sorption remained the dominant process. The current paper reanalyzes the data using a threesite sorption model to explore the influence of slow sorption processes.

\section{MATHEMATICAL MODEL}

A three-site model was developed to conceptualize three different types of sorption sites. Its purpose was to simulate (i) instantaneous exchange between solution and soil in the equilibrium phase; (ii) slow but reversible binding on nonequilibrium sorption sites characterized by a first-order sorption and desorption reaction with identical rate constant $k_{\mathrm{des}}$; and (iii) slow movement from nonequilibrium sites to irreversible sites, using the rate constant $k_{\text {irr }}$ (Figure 1 ). The model does not include degradation as the studies only lasted three days and Celis and Koskinen ${ }^{10,11}$ disregarded the process in the analysis of their results. All pesticide masses are in $\mu \mathrm{g}$, and all volumes are in $\mathrm{mL}$.

The quantity of pesticide sorbed at equilibrium sites $\left(X_{\text {eq }} ; \mu \mathrm{g}\right.$ $\mathrm{g}^{-1}$ ) was derived using the following equation:

$$
X_{\mathrm{eq}}=K_{\mathrm{feq}} C_{\mathrm{aq}}{ }^{1 / n}
$$

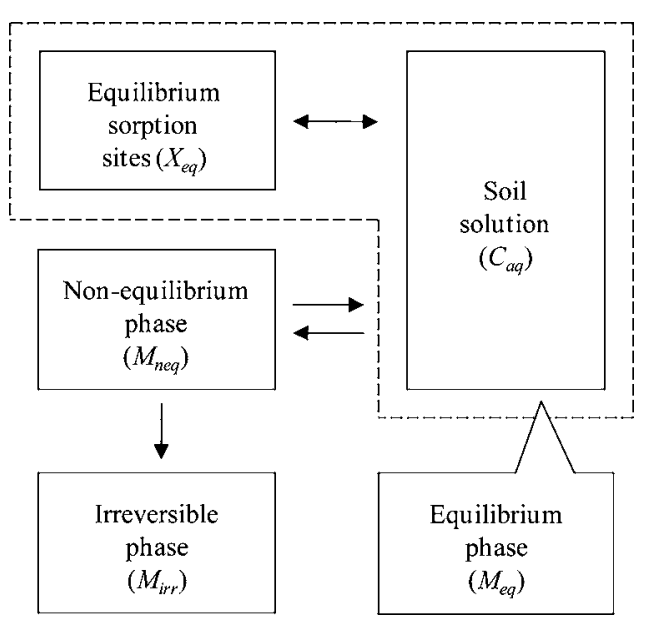

Figure 1. Schematic representation of the three-site model. The equilibrium phase $\left(M_{\mathrm{eq}}\right)$ includes both the concentration $\left(\mu \mathrm{g} \mathrm{mL}^{-1}\right)$ of pesticide in the soil solution $\left(C_{\mathrm{aq}}\right)$ and the pesticide sorbed $\left(\mu \mathrm{g} \mathrm{g}^{-1}\right)$ at equilibrium sites $\left(X_{\mathrm{eq}}\right)$.

where $K_{\mathrm{feq}}$ is the equilibrium Freundlich sorption distribution coefficient $\left(\mathrm{mL} \mathrm{g}^{-1}\right)$ and $1 / n$ is the Freundlich exponent (unitless), describing sorption nonlinearity with increasing solute concentration. The Taylor expansion is used to estimate $C_{\mathrm{aq}}\left(\mu \mathrm{g} \mathrm{mL}^{-1}\right)$; iteration in the Freundlich equation is used to solve the mass balance.

$X_{\text {eq }}$ is then used to determine the mass of pesticide in the equilibrium phase $\left(M_{\mathrm{eq}}\right)$ :

$$
M_{\text {eq }}=\left(V_{\text {sol }} C_{\text {aq }}\right)+\left(M_{\text {soil }} X_{\text {eq }}\right)
$$

where the parameters $V_{\text {sol }}$ and $M_{\text {soil }}$, respectively, refer to the volume of solution $(\mathrm{mL})$ and mass of soil $(\mathrm{g})$ used in the experimental system. In order to calculate the masses of pesticide in the nonequilibrium $\left(M_{\text {neq }}\right)$ and irreversible phases $\left(M_{\text {irr }}\right)$ of sorption $(\mu \mathrm{g})$, the following differential equations were used:

$$
\begin{aligned}
& \frac{\mathrm{d} M_{\text {neq }}}{\mathrm{d} t}=\left(k_{\mathrm{des}} F_{\mathrm{ne}} K_{\mathrm{feq}} C_{\mathrm{aq}}{ }^{1 / n}\right)-\left(k_{\mathrm{des}} M_{\mathrm{neq}}\right)-\left(k_{\mathrm{irr}} M_{\text {neq }}\right) \\
& \frac{\mathrm{d} M_{\mathrm{irr}}}{\mathrm{d} t}=k_{\text {irr }} M_{\text {neq }}
\end{aligned}
$$

where $k_{\text {des }}$ is the desorption or exchange rate $\left(\mathrm{d}^{-1}\right), F_{\text {ne }}$ is the ratio of sorption in the nonequilibrium phase to sorption in the equilibrium phase (unitless), and $k_{\text {irr }}$ is the rate of irreversible sorption $\left(d^{-1}\right)$.

The experimental setup necessitates the three-site model to be applied four times per pesticide in order to estimate the behavior of ${ }^{12} \mathrm{C}$ and ${ }^{14} \mathrm{C}$ isotopes both individually during the sorption phase and then simultaneously after supernatant exchange, for both initially ${ }^{12} \mathrm{C}$ samples (tube A) and initially ${ }^{12} \mathrm{C}$ and ${ }^{14} \mathrm{C}$ samples (tube B) (Figure 2).

The following equations were used to calculate the isotope masses transferred between the two corresponding tubes at supernatant exchange:

In tube A:

$$
\begin{aligned}
& M_{\text {eq }}{ }^{12} \mathrm{C}=M_{\text {eq (tubeA })}-V_{\text {rem }} C_{\text {aq }(\text { tubeA })} \\
& M_{\text {eq }}{ }^{14} \mathrm{C}=V_{\text {add }} C_{\text {aq (tubeB })}
\end{aligned}
$$




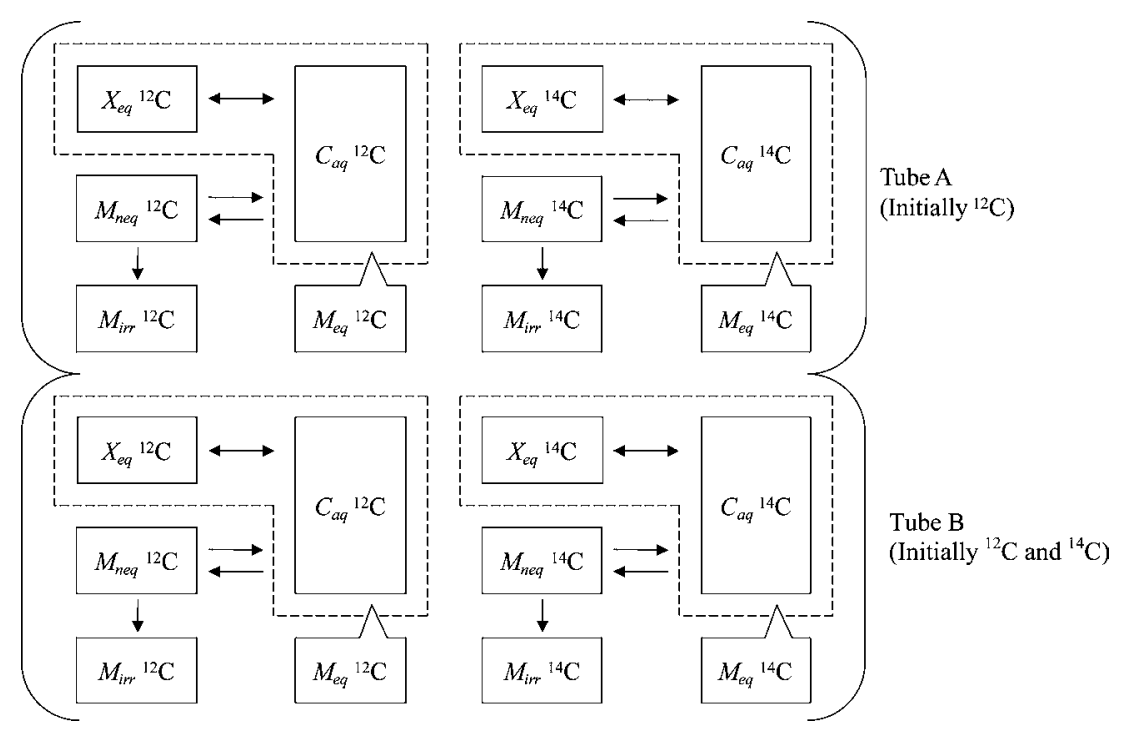

Figure 2. Expanded schematic representation of the three-compartment model to account for the fact that (i) tubes A initially contained only ${ }^{12} \mathrm{C}$ pesticide and tubes $\mathrm{B}$ initially contained both ${ }^{12} \mathrm{C}$ - and ${ }^{14} \mathrm{C}$-pesticide; and (ii) after supernatant exchange tubes $\mathrm{A}$ and $\mathrm{B}$ both contained ${ }^{12} \mathrm{C}$ and ${ }^{14} \mathrm{C}$ isotopes. The basis for simulation of tubes $\mathrm{A}$ and $\mathrm{B}$ is identical, but these differ in the initial mass of each isotope used as input to the model.

In tube $\mathrm{B}$ :

$$
\begin{aligned}
& M_{\text {eq }}{ }^{12} \mathrm{C}=V_{\text {add }} C_{\text {aq(tubeA })} \\
& M_{\text {eq }}{ }^{14} \mathrm{C}=M_{\text {eq(tubeB })}-V_{\text {rem }} C_{\text {aq(tubeB })}
\end{aligned}
$$

where $V_{\text {rem }}$ is the original volume of supernatant removed from each tube at supernatant exchange $(\mathrm{mL})$, and $V_{\text {add }}$ is the added volume of supernatant from the other tube $(\mathrm{mL})$ that replaces the original supernatant.

In the model, the equilibrium sorption reaction is assumed to be instantaneous. As this reaction does not differentiate between ${ }^{12} \mathrm{C}$ - and ${ }^{14} \mathrm{C}$-pesticide, the Freundlich equation is applied to both tubes simultaneously. This necessitates calculating the fraction of total pesticide isotopes represented by ${ }^{14} \mathrm{C}$-pesticide in tube $\mathrm{A}$ and tube $\mathrm{B}\left(\mathrm{F}_{14 \mathrm{C}}\right)$ :

$$
F_{14 \mathrm{C}}=\frac{M_{\mathrm{eq}}{ }^{14 \mathrm{C}}}{M_{\mathrm{eq}}}
$$

where $M_{\mathrm{eq}}{ }^{14 \mathrm{C}}$ refers only to the mass of ${ }^{14} \mathrm{C}$-pesticide in the equilibrium phase $(\mu \mathrm{g}) . F_{14 C}$ is calculated by the model at each time step. The fraction of ${ }^{14} \mathrm{C}$-pesticide is considered in the flow from the equilibrium to the nonequilibrium phases. In order to account for this in the model, the following equations are used to calculate the concentrations of ${ }^{12} \mathrm{C}$ - and ${ }^{14} \mathrm{C}$ pesticide in solution for tubes $\mathrm{A}$ and $\mathrm{B}$, respectively.

In tube A:

$$
\begin{aligned}
& C_{\mathrm{aq}}{ }^{12 \mathrm{C}}=\left(1-F_{14 \mathrm{C}(\text { tubeA })}\right) C_{\text {aq(tubeA })} \\
& C_{\mathrm{aq}}{ }^{14 \mathrm{C}}=F_{14 \mathrm{C}(\text { tubeA })} C_{\mathrm{aq}(\text { tubeA })}
\end{aligned}
$$

In tube $\mathrm{B}$ :

$$
\begin{aligned}
& C_{\mathrm{aq}}{ }^{12 \mathrm{C}}=\left(1-F_{14 \mathrm{C}(\text { tubeB })}\right) C_{\text {aq(tubeB })} \\
& C_{\mathrm{aq}}{ }^{14 \mathrm{C}}=F_{14 \mathrm{C}(\text { tubeB })} C_{\mathrm{aq}(\text { tubeB })}
\end{aligned}
$$

For output purposes, the specific activity $\left(\mathrm{Bq} \mu \mathrm{g}^{-1}\right)$ was included as a parameter in the model, and was used to convert the concentration in the aqueous phase expressed within the model $\left(\mu \mathrm{g} \mathrm{mL}^{-1}\right)$ to that reported in the original study $(\mathrm{Bq}$ $\left.\mathrm{mL}^{-1}\right)$.

The model has three unknown parameters that describe the sorption behavior of the pesticides: (i) the ratio of nonequilibrium sorption to equilibrium sorption $\left(F_{\text {ne }}\right)$, (ii) the desorption rate $\left(k_{\text {des }}\right)$, and (iii) the rate of irreversible sorption $\left(k_{\text {irr }}\right)$. The Freundlich distribution coefficient $\left(K_{\text {feq }}\right)$ and the Freundlich exponent $(1 / n)$ were measured by Celis and Koskinen ${ }^{10,11}$ by independent Freundlich sorption studies for all studied pesticide/soil systems, so these values were included in the three-site model. The three unknown parameters were optimized using the Marquardt method and weighted leastsquares. The optimization was carried out using the default settings of ModelMaker software (version 4.0), using a convergence change of 0.1 and 50 convergence steps. The default Marquardt settings were as follows: initial lambda of 0.01 ; minimum change of $1 \times 10^{-5}$; and fractional change of 0.01 . Individual weighting was used with a data error fraction of 1.

Lastly, the model fit to the data for all pesticide and soil systems was quantified statistically using the following model efficiency (ME) equation:

$$
\mathrm{ME}=1-\frac{\sum_{i=1}^{n}\left(C_{i}-O_{i}\right)^{2}}{\sum_{i=1}^{n}\left(O_{i}-\bar{O}_{i}\right)^{2}}
$$

where $n$ is the total number of observations, $O_{i}$ is the $i$ th observed value (with $i=1,2, \ldots, n$ ), $C_{i}$ is the $i$ th value calculated by the model, and $\bar{O}$ is the mean of all observed values. The ME compares the sum of squared differences between the calculated and observed data with the variability in the observed data. The ME may range from minus infinity to +1 , and larger absolute values indicate a better agreement between the measured data and modeled values.

\section{RESULTS AND DISCUSSION}

The three-site model was applied to describe the data obtained from five of the pesticide/soil systems Celis and Koskinen ${ }^{10,11}$ studied in their two isotope-exchange papers: (i) imidaclopridguanidine/Hanford soil, (ii) imidacloprid-guanidine/Drummer 
Table 1. Measured and Optimized Parameter Values of the Model Used To Describe Celis and Koskinen's ${ }^{10,11}$ IsotopeExchange Data for the Five Pesticide/Soil Systems Studied

\begin{tabular}{|c|c|c|c|c|c|}
\hline \multirow[b]{2}{*}{ parameter } & \multicolumn{5}{|c|}{ parameter values per pesticide/soil system } \\
\hline & $\begin{array}{l}\text { imidacloprid-guanidine/ } \\
\text { Hanford }\end{array}$ & $\begin{array}{l}\text { imidacloprid-guanidine/ } \\
\text { Drummer }\end{array}$ & $\begin{array}{l}\text { imidacloprid/ } \\
\mathrm{SiCL}^{a}\end{array}$ & $\begin{array}{l}\text { imidacloprid-urea/ } \\
\operatorname{SiCL}^{a}\end{array}$ & $\begin{array}{l}\text { imidacloprid- } \\
\text { guanidine } / L S^{b}\end{array}$ \\
\hline \multicolumn{6}{|l|}{ measured values } \\
\hline $\begin{array}{l}K_{\text {feq }}(\text { Freundlich distribution } \\
\text { coefficient })\end{array}$ & 6.75 & 42.2 & 6.70 & 3.10 & 3.57 \\
\hline $1 / n$ (Freundlich exponent) & 0.92 & 0.83 & 0.87 & 0.88 & 1.05 \\
\hline $\begin{array}{l}{ }^{12} \mathrm{C}(\mu \mathrm{g}) \\
\end{array}$ & 3.15 & 3.15 & 3.15 & 3.15 & 3.15 \\
\hline $\begin{array}{l}\text { initial mass of tube } \mathrm{B} \text {, initially } \\
{ }^{14} \mathrm{C}(\mu \mathrm{g})\end{array}$ & 3.15 & 3.15 & 3.15 & 3.15 & 3.15 \\
\hline mass of soil $(\mathrm{g})$ & 0.5 & 0.5 & 2.0 & 2.0 & 2.0 \\
\hline specific activity $\left(\mathrm{Bq} \mu \mathrm{g}^{-1}\right)$ & 246.03 & 246.03 & 239.68 & 239.68 & 239.68 \\
\hline $\begin{array}{l}\text { volume of supernatant removed } \\
(\mathrm{mL})\end{array}$ & 8.0 & 8.0 & 7.0 & 7.0 & 7.0 \\
\hline $\begin{array}{l}\text { volume of supernatant replaced } \\
(\mathrm{mL})\end{array}$ & 8.0 & 8.0 & 7.0 & 7.0 & 7.0 \\
\hline $\begin{array}{l}\text { total volume of shaking solution } \\
(\mathrm{mL})\end{array}$ & 10.0 & 10.0 & 10.0 & 10.0 & 10.0 \\
\hline \multicolumn{6}{|l|}{ optimized values } \\
\hline$F_{\text {ne }}^{c}($ ratio $)$ & $0.16(0.10)$ & $0.049(0.010)$ & $1.07(0.20)$ & $0.81(0.072)$ & $2.80(0.23)$ \\
\hline$k_{\text {des }}^{c}\left(\mu \mathrm{g} \mathrm{day}^{-1}\right)$ & $0.016(0.017)$ & $0.090(0.11)$ & $0.034(0.013)$ & $0.037(0.0070)$ & $0.027(0.0040)$ \\
\hline \multicolumn{6}{|l|}{ fixed values } \\
\hline$k_{\text {irr }}\left(\mu \mathrm{g} \mathrm{day}^{-1}\right)$ & 0.0 & 0.0 & 0.0 & 0.0 & 0.0 \\
\hline
\end{tabular}

soil, (iii) imidacloprid/SiCL (silty clay loam) soil, (iv) imidacloprid-urea/SiCL soil, and (v) imidacloprid-guanidine/ LS (loamy sand) soil.

The measured and optimized constants used to parametrize the model are given in Table 1 . Attempts to include $k_{\text {irr }}$ in the optimization with $F_{\text {ne }}$ and $k_{\text {des }}$ resulted in the model being unable to generate robust $F_{\text {ne }}, k_{\text {des }}$, and $k_{\text {irr }}$ values because optimization errors exceeded the parameter estimates calculated by the model. Thus, it was necessary to fix the parameter for irreversible sorption $\left(k_{\text {irr }}\right)$ to zero, and effectively use a twosite model (using only equilibrium and nonequilibrium sorption), to generate an accurate simulation of Celis and Koskinen's ${ }^{10,11}$ isotope-exchange data.

The model fit is plotted against the isotope-exchange data measured by Celis and Koskinen ${ }^{10,11}$ for all pesticide/soil systems in Figures 3 and 4 . Visually, the model with $k_{\text {irr }}$ set to zero is able to describe the measured isotope-exchange data very well. The model efficiency (ME) equation was used to quantify this for the five pesticide/soil systems studied (Table 2 ). Despite the fact that optimization errors generated by ModelMaker are generally quite high (Table 1), the calculated model efficiencies are very close to one for all models tested $(\geq 0.984)$. Thus, although there is some uncertainty concerning the accuracy of the optimized parameter values, the two-site model gives a good overall fit to the isotope-exchange data.

The model also confirms and explains the asymmetry observed between measured data from initially ${ }^{12} \mathrm{C}$ and ${ }^{14} \mathrm{C}$ samples and relative deviation from their respective expected radioactivity lines. The measured data for initially ${ }^{12} \mathrm{C}$ samples is always closer to the expected radioactivity than for the samples containing both ${ }^{12} \mathrm{C}$ - and ${ }^{14} \mathrm{C}$-labeled pesticide (Figures 3 and 4). This is because equilibrium was not reached during the $24 \mathrm{~h}$ sorption phase. Without attaining equilibrium, sorption is still the dominant process, thus comparatively, desorption of ${ }^{14} \mathrm{C}$-pesticide from the initially ${ }^{14} \mathrm{C}$ sample occurs
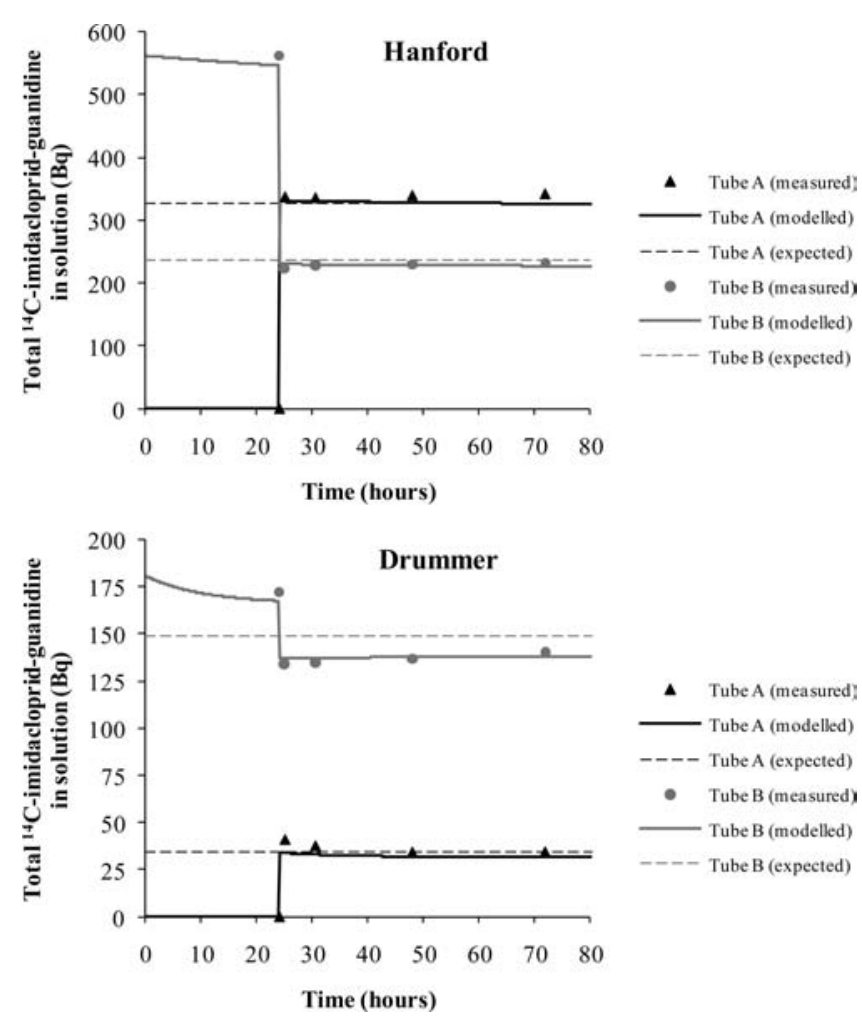

Figure 3. Isotope exchange of imidacloprid-guanidine in the Hanford and Drummer soils (Figure 6, Celis and Koskinen ${ }^{10}$ ). The dotted lines represent the radioactivity in solution that the measured radioactivities are expected to reach if there is perfect exchange, i.e., if sorption is a fully reversible process.

more slowly. The importance of slow sorption kinetics has been documented by a number of authors; ${ }^{13-15}$ thus, this process 

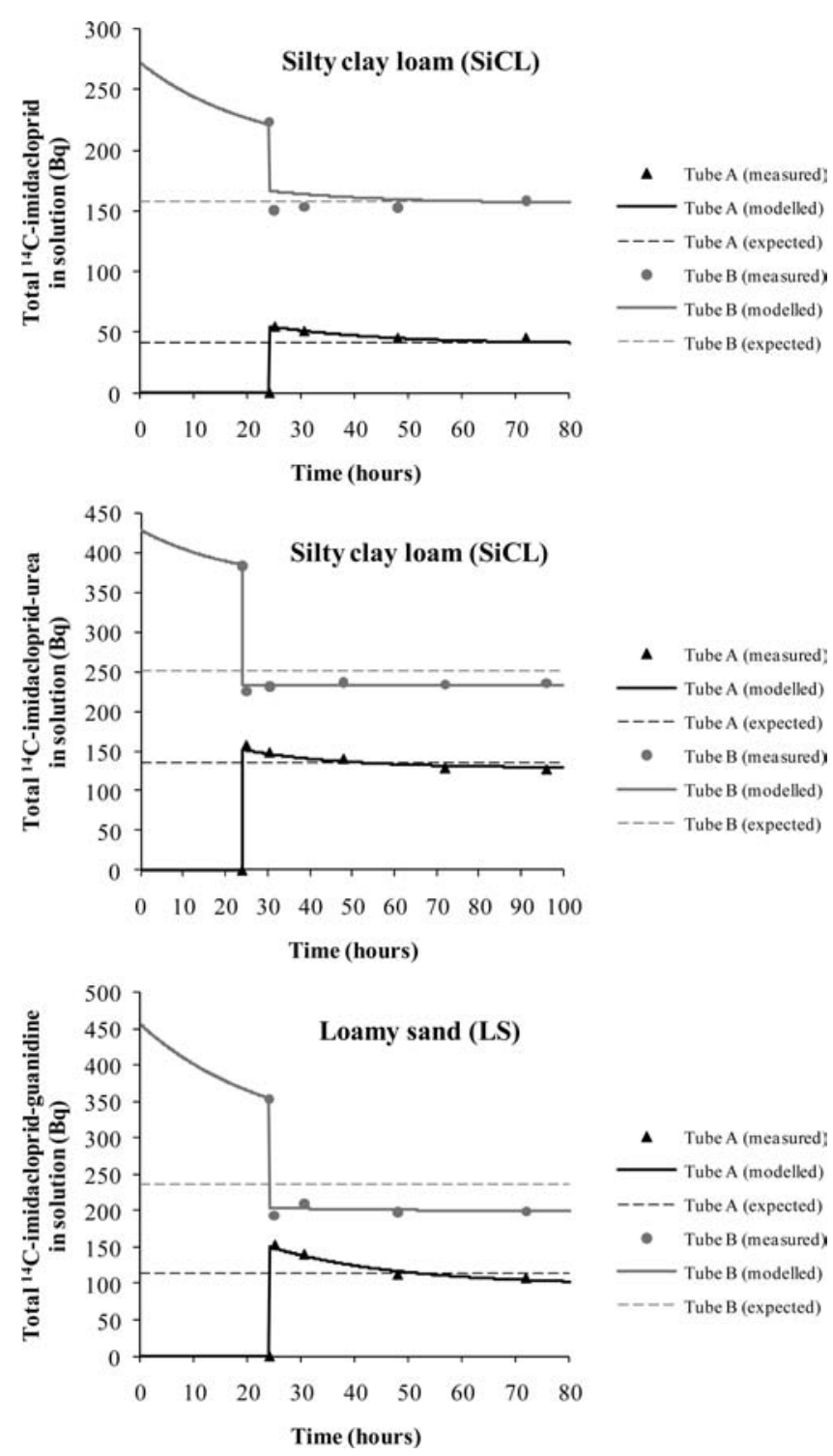

Figure 4. Isotope exchange in the three soil systems studied (Figure 2, Celis and Koskinen $\left.{ }^{11}\right)$. The dotted lines represent the radioactivity in solution that the measured radioactivities are expected to reach if there is perfect exchange, i.e., if sorption is a fully reversible process.

Table 2. Calculated Model Efficiency (ME)

\begin{tabular}{lc}
\multicolumn{1}{c}{ pesticide/soil system } & calcd model efficiency (ME) \\
imidacloprid-guanidine/Hanford soil $^{a}$ & 0.996 \\
imidacloprid-guanidine/Drummer soil $^{a}$ & 0.997 \\
imidacloprid/SiCL soil $^{b}$ & 0.984 \\
imidacloprid-urea/SiCL soil $^{b}$ & 0.997 \\
imidacloprid-guanidine LS soil $^{b}$ & 0.996 \\
${ }^{a}$ Data from Celis and Koskinen, ${ }^{10}$ Figure 6. ${ }^{b}$ Data from Celis and \\
Koskinen, ${ }^{11}$ Figure 2.
\end{tabular}

cannot be ignored in the interpretation of isotope-exchange studies.

The experimental design and the short time scales involved in the sorption and isotope-exchange phases restrict the ability to discriminate between slowly reversible and irreversible sorption. Thus, the fact that the two-site model describes the isotope-exchange data with $k_{\text {irr }}$ set to zero does not prove that irreversible sorption did not occur. Abundant evidence has

shown that attaining sorption equilibrium for many compounds may take days, months, or even years. ${ }^{14,16,17}$ Thus, carrying out isotope-exchange studies using longer sorption and isotopeexchange phases may augment the ability of isotope-exchange studies to differentiate between slowly reversible and irreversible sorption.

In summary, application of the two-site model to Celis and Koskinen's isotope-exchange data showed that the measurements obtained could be described using two sorption sites (instantaneous exchange and slow but reversible binding), and that this fit was much better than when irreversible sorption was included. Degradation may be another complicating process resulting in similar isotope-exchange behavior to that resulting from slow sorption, with greater deviation from the respective radioactivity lines in tube $\mathrm{B}$ compared to tube $\mathrm{A}$; however, as the studies were only three days long it is unlikely that degradation had any significant effect.

In most existing sorption models, irreversible binding is not included. Selecting an appropriate model to describe desorption profiles has however been reported to be system-specific. For example, Saffron et al. ${ }^{18}$ divided the desorption profile into three regimes: (i) a fast or instantaneous regime, where desorption occurs at rates not captured by the first few sampling points; (ii) a dynamic regime in which rates are well measured by the sampling scheme; and (iii) a slow regime where rates are slower than can be measured given the combination of data uncertainty and duration of sampling. They found that although naphthalene desorption was best described by two regimes, all three were required to adequately describe the desorption behavior of atrazine. Furthermore, Johnson et al. ${ }^{19}$ compared six modeling approaches in their ability to describe phenanthrene desorption data: (i) a threeparameter kinetic model, (ii) a five-parameter kinetic model, (iii) a gamma-distribution model, (iv) a one-parameter porediffusion model, (v) a two-parameter pore-diffusion model, and (vi) a three-parameter biphasic polymer diffusion model. They concluded that all desorption profiles were at least biphasic and that models composed of two regimes provide a good basis for describing desorption profiles. Further work is required to develop long-term experiments that are able to effectively differentiate between slowly reversible sorption and irreversible sorption.

\section{AUTHOR INFORMATION}

\section{Corresponding Author}

*E-mail: laurasuddaby@gmail.com. Tel: +44 (0) 1904462166.

\section{Notes}

The authors declare no competing financial interest.

\section{ACKNOWLEDGMENTS}

EPSRC and Syngenta Ltd are gratefully acknowledged for funding this research.

\section{REFERENCES}

(1) Spark, K. M.; Swift, R. S. Effect of soil composition and dissolved organic matter on pesticide sorption. Sci. Total Environ. 2002, 298 (13), 147-161.

(2) Boivin, A.; Cherrier, R.; Schiavon, M. A comparison of five pesticides adsorption and desorption processes in thirteen contrasting field soils. Chemosphere 2005, 61, 668-676.

(3) Wang, P.; Keller, A. A. Sorption and desorption of atrazine and diuron onto water dispersible soil primary size fractions. Water Res. 2009, 43, 1448-1456. 
(4) Huang, W.; Yu, H.; Weber, W. J., Jr. Hysteresis in the sorption and desorption of hydrophobic organic contaminants by soils and sediments: 1. A comparative analysis of experimental protocols. J. Contam. Hydrol. 1998, 31 (1-2), 129-148.

(5) Dec, J.; Haider, K.; Schäffer, A.; Fernandes, E.; Bollag, J. M. Use of a silylation procedure and ${ }^{13} \mathrm{C}$-NMR spectroscopy to characterise bound and sequestered residues of cyprodinil in soil. Environ. Sci. Technol. 1997, 31, 2991-2997.

(6) Mordaunt, C. J.; Gevao, B.; Jones, K. C.; Semple, K. T. Formation of non-extractable pesticide residues: observations on compound differences, measurement and regulatory issues. Environ. Pollut. 2005, 133, 25-34.

(7) Wanner, U.; Führ, F.; deGraaf, A. A.; Burauel, P. Characterization of non-extractable residues of the fungicide dithianon in soil using ${ }^{13} \mathrm{C} /{ }^{14} \mathrm{C}$-labelling techniques. Environ. Pollut. 2005, 133 (1), 35-41.

(8) Huang, W.; Weber, W. J. A distributed reactivity model for sorption by soils and sediments. 10. Relationships between desorption, hysteresis, and the chemical characteristics of organic domains. Environ. Sci. Technol. 1997, 31 (9), 2562-2569.

(9) Northcott, G. L.; Jones, K. C. Experimental approaches and analytical techniques for determining organic compound bound residues in soil and sediment. Environ. Pollut. 2000, 108 (1), 19-43.

(10) Celis, R.; Koskinen, W. C. An isotopic exchange method for the characterisation of the irreversibility of pesticide sorption-desorption in soil. J. Agric. Food Chem. 1999, 47, 782-790.

(11) Celis, R.; Koskinen, W. C. Characterisation of pesticide desorption from soil by the isotopic exchange technique. Soil Sci. Soc. Am. J. 1999, 63, 1659-1666.

(12) Sander, M.; Pignatello, J. J. An isotope exchange technique to assess mechanisms of sorption hysteresis applied to naphthalene in kerogenous organic matter. Environ. Sci. Technol. 2005, 39 (19), 7476-7484.

(13) Altfelder, S.; Streck, T. Capability and limitations of first-order and diffusion approaches to describe long term sorption of chlorotoluron in soil. J.Contam. Hydrol. 2006, 86, 279-298.

(14) Ball, W. P.; Roberts, P. V. Long-term sorption of halogenated organic chemicals by aquifer material. 2. Intraparticle diffusion. Environ. Sci. Technol. 1991, 25 (7), 1237-1249.

(15) Pignatello, J. J.; Xing, B. Mechanisms of slow sorption of organic chemicals to natural particles. Environ. Sci. Technol. 1996, 30 (1), 111.

(16) Renaud, F. G.; Leeds-Harrison, P. B.; Brown, C. D.; van Beinum, W. Determination of time-dependent partition coefficients for several pesticides using diffusion theory. Chemosphere 2004, 57, 15251535.

(17) Beulke, S.; Brown, C. D.; Fryer, C. J.; van Beinum, W. Influence of kinetic sorption and diffusion on pesticide movement through aggregated soils. Chemosphere 2004, 57, 481-490.

(18) Saffron, C. M.; Park, J.-H.; Dale, B. E.; Voice, T. C. Kinetics of contaminant desorption from soil: comparison of model formulations using the Akaike information criterion. Environ. Sci. Technol. 2006, 40 (24), 7662-7667.

(19) Johnson, M. D.; Keinath, T. M.; Weber, W. J. A distributed reactivity model for sorption by soils and sediments. 14. Characterization and modeling of phenanthrene desorption rates. Environ. Sci. Technol. 2001, 35 (8), 1688-1695. 